\title{
Real-Time Assessment of Tissue Hypoxia In Vivo with Combined Photoacoustics and High-Frequency Ultrasound
}

Marco Gerling1 ${ }^{*}$, Ying Zhao $2,3^{*}$, Salvatore Nania ${ }^{3}$, K. Jessica Norberg 3 , Caroline S. Verbeke ${ }^{4}$, Benjamin Englert ${ }^{1}$, Raoul V. Kuiper 6 , Åsa Bergström ${ }^{1}$, Moustapha Hassan ${ }^{5}$, Albrecht Neesse7 ${ }^{7}$ J. Matthias Löhr1,3, Rainer L. Heuchel1,3凶

1. Center of Biosciences, Department of Biosciences and Nutrition, Karolinska Institutet, Stockholm, Sweden;

2. StratCan Preclinical Cancer Test Facility, Karolinska Institutet, Stockholm, Sweden;

3. Pancreas Research Laboratory, CLINTEC, Karolinska Institutet, Stockholm, Sweden;

4. Division of Pathology, Karolinska Institutet, Stockholm, Sweden;

5. Clinical Research Centre, Karolinska University Hospital-Huddinge, Stockholm, Sweden;

6. Core Facility for Morphologic Phenotype Analysis, Clinical Research Center, Karolinska Institutet, Stockholm, Sweden;

7. Department of Gastroenterology, Endocrinology and Metabolism, Philipps University Marburg, Marburg, Germany.

* equal contribution.

$\triangle$ Corresponding author: Rainer Heuchel, PhD. Pancreatic Cancer Research Laboratory CLINTEC, Karolinska Institutet, Hälsovägen 7, SE-141 86 Stockholm, Sweden. rainer.heuchel@ki.se.

(c) Ivyspring International Publisher. This is an open-access article distributed under the terms of the Creative Commons License (http://creativecommons.org/ licenses/by-nc-nd/3.0/). Reproduction is permitted for personal, noncommercial use, provided that the article is in whole, unmodified, and properly cited.

Received: 2013.10.28; Accepted: 2014.02.04; Published: 2014.03.18

\begin{abstract}
Purpose: In preclinical cancer studies, non-invasive functional imaging has become an important tool to assess tumor development and therapeutic effects. Tumor hypoxia is closely associated with tumor aggressiveness and is therefore a key parameter to be monitored. Recently, photoacoustic (PA) imaging with inherently co-registered high-frequency ultrasound (US) has reached preclinical applicability, allowing parallel collection of anatomical and functional information. Dual-wavelength PA imaging can be used to quantify tissue oxygen saturation based on the absorbance spectrum differences between hemoglobin and deoxyhemoglobin.

Experimental Design: A new bi-modal PA/US system for small animal imaging was employed to test feasibility and reliability of dual-wavelength PA for measuring relative tissue oxygenation. Murine models of pancreatic and colon cancer were imaged, and differences in tissue oxygenation were compared to immunohistochemistry for hypoxia in the corresponding tissue regions.

Results: Functional studies proved feasibility and reliability of oxygenation detection in murine tissue in vivo. Tumor models exhibited different levels of hypoxia in localized regions, which positively correlated with immunohistochemical staining for hypoxia. Contrast-enhanced imaging yielded complementary information on tissue perfusion using the same system.

Conclusion: Bimodal PA/US imaging can be utilized to reliably detect hypoxic tumor regions in murine tumor models, thus providing the possibility to collect anatomical and functional information on tumor growth and treatment response live in longitudinal preclinical studies.
\end{abstract}

Key words: Photoacoustics, High-Frequency Ultrasound, Hypoxia.

\section{Introduction}

Photoacoustic (PA) imaging is a rapidly evolving technique based on the optical excitation of molecules by laser pulses resulting in thermoelastic waves that can be detected by ultrasound (US) receivers (for a concise review, please refer to Wang and $\mathrm{Hu}$, Science, 2012(1)). As an improvement to traditional optical 
imaging, PA is able to detect light-absorbing molecules deep inside tissues with far superior resolution (1). While various exogenous contrast agents are currently under development (2-4), PA can exploit excitable endogenous contrast such as hemoglobin to create images of living tissues (5-7).

Absorption spectra of hemoglobin differ with oxygenation and therefore offer the additional possibility of functional analysis (8): Dual-wavelength PA can be used to separately measure tissue concentration of hemoglobin $\left(\mathrm{HbO}_{2}\right)$ and deoxyhemoglobin $(\mathrm{Hb})$ and as such to calculate focal tissue oxygen saturation $(9,10)$. By imaging $\mathrm{HbO}_{2}$ in blood vessels, three dimensional blood vasculature networks have been studied in superficial tissues such as subcutaneous xenografts, rodent ears, as well as endoscopically in rat intestine $(9,11,12)$. In the latter study, PA endoscopy was elegantly combined with endoscopic US, resulting in an intricate combination of anatomic and functional information (12).

Recent efforts have led to the successful combination of conventional US- and PA-systems that allow for concise co-registration and live overlay of high-resolution anatomical data from US (mechanical contrast) and PA data (optical contrast) (13-15). Such systems facilitate unequivocal identification of anatomical structures and - depending on the selected PA wavelengths - functional information such as the degree of oxygenation.

Tissue hypoxia has been recognized as a hallmark of solid cancers and is frequently associated with poor prognosis and increased tumor aggressiveness $(16,17)$. Therapeutic alterations of the oxygen levels in tumors and the tumor microenvironment are therefore promising approaches that are being translated into clinical application $(18,19)$.

Modern animal models of human cancers can mimic their human counterpart to an ever-increasing extent, fuelling optimism regarding preclinical assessment of novel therapeutics with high clinical predictability (20-22). Accordingly, preclinical test facilities are being developed worldwide. As genetically engineered mouse models are labor- and cost-intensive, live-imaging modalities to follow tumor development longitudinally are urgently needed. Ideally, such imaging modalities combine information about tumor volume and anatomy with functional data.

With the emergence of PA, efforts were directed to image oxygenation levels in vivo. In pioneering studies, the group of Lihong Wang provided compelling proof-of-principle of hypoxia measurements in rodent brain tumors using spectral photoacoustic tomography (SPAT) $(23,24)$. Intriguing images of oxygen saturation at high resolution were also presented by other groups in further experiments based on photoacoustic microscopy (PAM) (5). The latter PA technique competes with e.g. multiphoton microscopy in terms of resolution, yet reaches constraints when in comes to imaging of internal organs in orthotopic models due to depth limitations (15). Both approaches did not yet support co-registration with conventional US, while a comprehensive comparison with structural data, Doppler data, or intravenous contrast as a measure for perfusion would greatly contextualize PA measurements. An elegant approach in combining US with PA to measure hypoxia in subcutaneous tumors was pursued in a recent study by Shao et al., in which the authors utilized intravenously injected methylene blue as exogenous contrast (25).

The need for an exogenous contrast agent can be circumvented by the use of dual-wavelength PA. In the present study, we sought to address the feasibility of investigating tumor development and tumor oxygenation in a label-free approach with 3-dimensional PA imaging in parallel to conventional B-mode US. We provided evidence that this combined system can be used to reliably assess tissue oxygenation in murine organs while yielding structural information at high resolution. We subsequently used the system to assess hypoxia and tumor blood flow in murine models of pancreatic and colon cancer as examples of intra-abdominal tumors, and observed a positive correlation of PA data with immunohistochemical evidence of hypoxia.

\section{Results}

\section{Real-time photoacoustic imaging detects alterations in oxygen levels in murine organs}

To test the ability of the PA system to detect oxygenation changes, mice anaesthetized with isoflurane $(1.5 \% \mathrm{v} / \mathrm{v})$ and supplied with medical air $(21 \%$ $\mathrm{v} / \mathrm{v} \mathrm{O}_{2}, 2 \mathrm{~L} / \mathrm{min}$ ) underwent abdominal US with PA co-registration at dual wavelengths (Figure 1, and Additional file 1: Suppl. video 1). Baseline levels of oxygenation were assessed over 2 minutes. Thereafter, oxygen supply was increased to $100 \% \mathrm{v} / \mathrm{v} \mathrm{O}_{2}$ (2L/min). Oxygenation levels were registered live over 8 minutes, before changing back to medical air. Liver parenchyma and the gastric wall were selected as regions of interest for PA imaging, and oxygen levels were plotted over time. PA imaging showed an increase of tissue oxygenation for both regions upon administration of pure oxygen, while this increase was more rapid and more pronounced for liver parenchyma than for the gastric wall. Adjusting to ambient air led to normalization of oxygen levels as assessed by PA imaging after an initial drop below 
starting levels.

\section{Detection of tumor hypoxia in pancreatic isografts and murine colon cancer, and correlation with histologic assessment}

Next, we employed a syngeneic pancreatic cancer model based on injection of murine pancreatic adenocarcinoma cells (derived from the Kras $^{L S L-G 12 D /+} ; p 53^{R 172 H /+} ; P d x 1-C r e$ model (26), hereafter referred to as KPC cells) subcutaneously and intra-

A

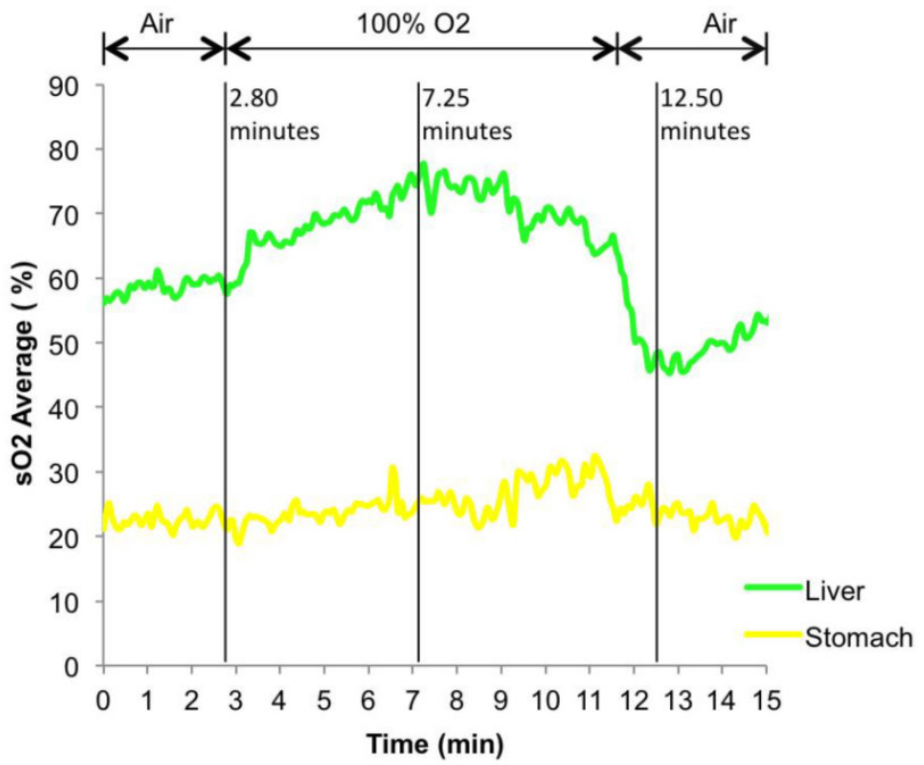

pancreatically. Two weeks post injection, tumors were scanned with PA and B-mode US in 3D. After image registration, pimonidazole was injected intraperitoneally for post-mortem immunohistochemical analysis of hypoxia (27).

KPC tumors at different stages were associated with different degrees of hypoxia. Figure 2A-C gives an example of a tumor with two separated hypoxic regions, which were identifiable on PA imaging. Figure 2D-F shows a PA example of a non-hypoxic smaller tumor with corresponding histology.

Figure I. Oxygen saturation in liver parenchyma and stomach wall during changes of oxygen supply. A) Relative $\mathrm{sO}_{2}$ (oxygen saturation) in liver parenchyma and stomach wall. Oxygen saturation is calculated as the percentage of oxygenated hemoglobin relative to total hemoglobin based on dual-wavelength PA imaging at 750 $\mathrm{nm} / 850 \mathrm{~nm}$. Gas supply was changed from air to $100 \% \mathrm{O}_{2}$ at 2.8 minutes, from $100 \% \mathrm{O}_{2}$ back to air at 11.5 minutes. B) Representative Oxy-Hemo photoacoustic images co-registered with greyscale B-mode images at $2.8,7.25,12.5$ minutes. The heat map represents oxygen saturation levels ranging from $100 \%$ (red) to $0 \%$ (dark blue). Black pixels equal the absence of signal above a defined threshold of $20 \%$ of maximum (see methods). Oxygen saturation is calculated as the percentage of oxygenated hemoglobin in relation to total hemoglobin.

B
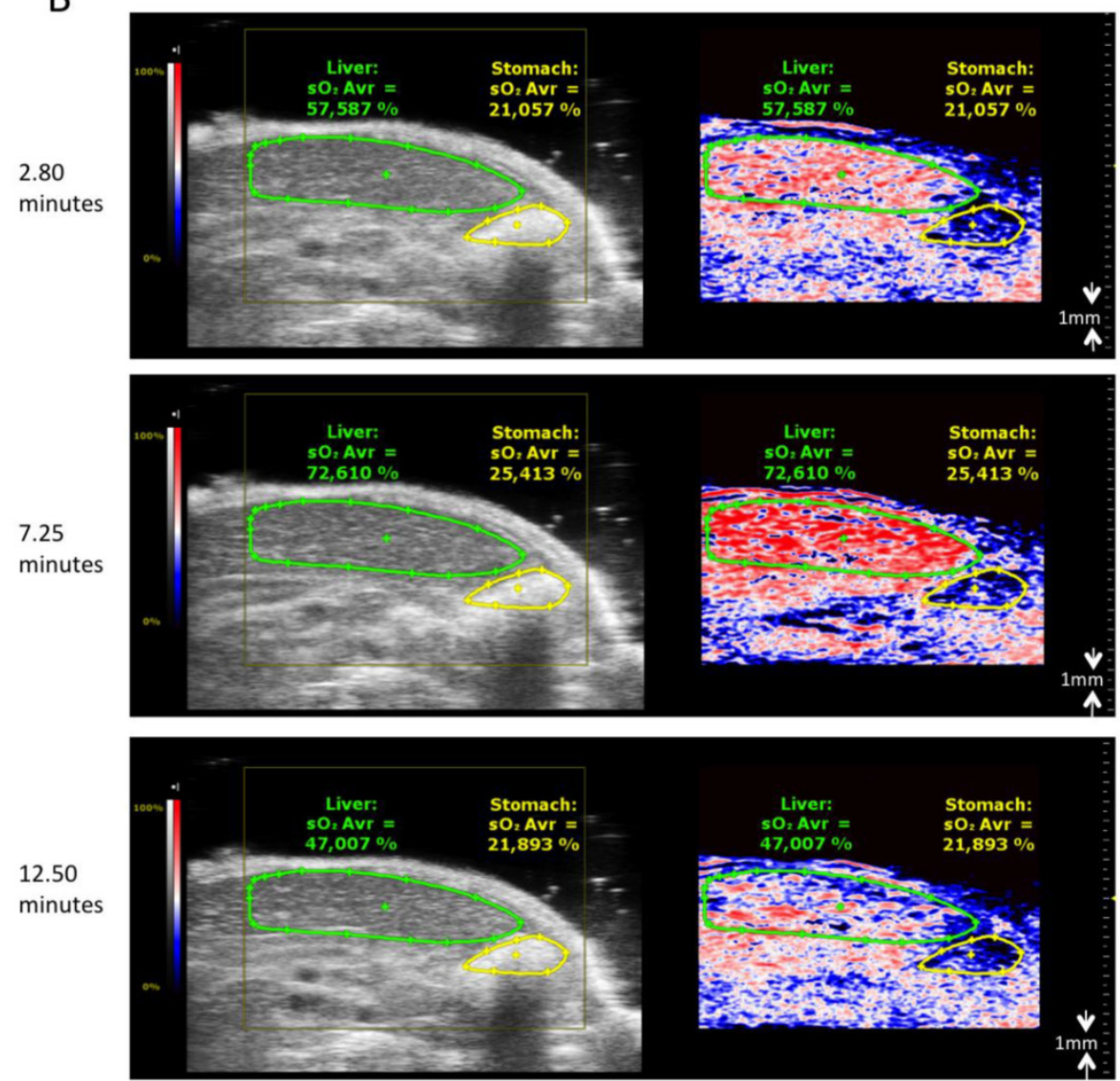
A

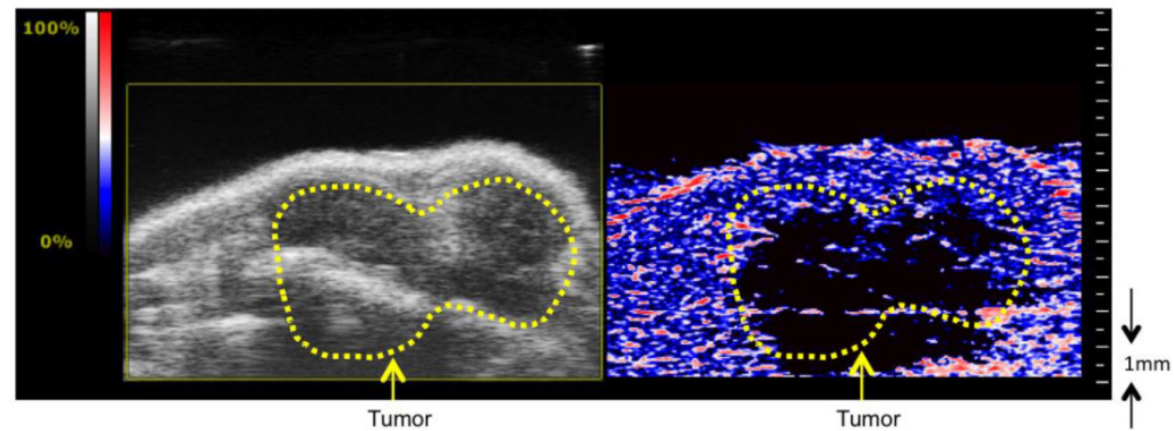

B

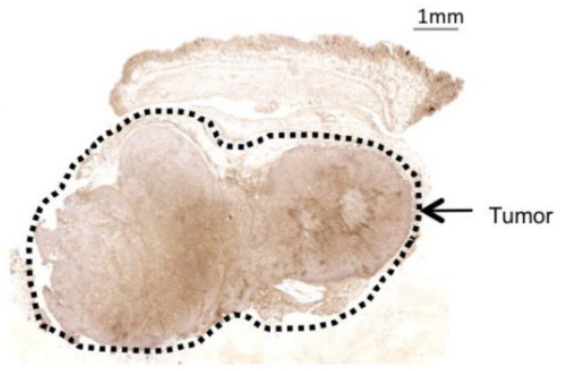

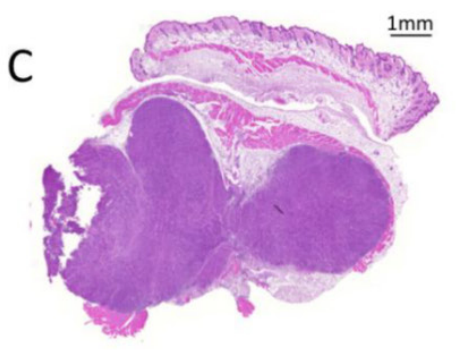

D

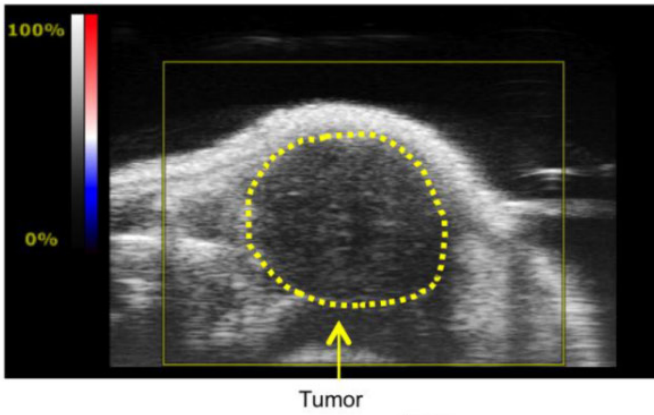

$\mathrm{E}$
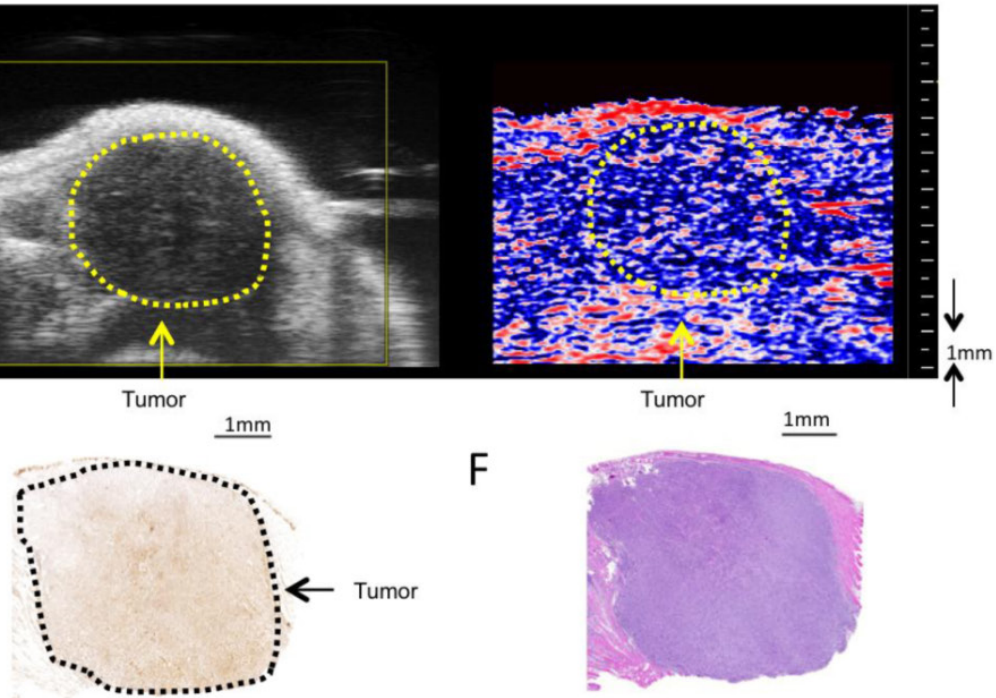

$\mathrm{F}$
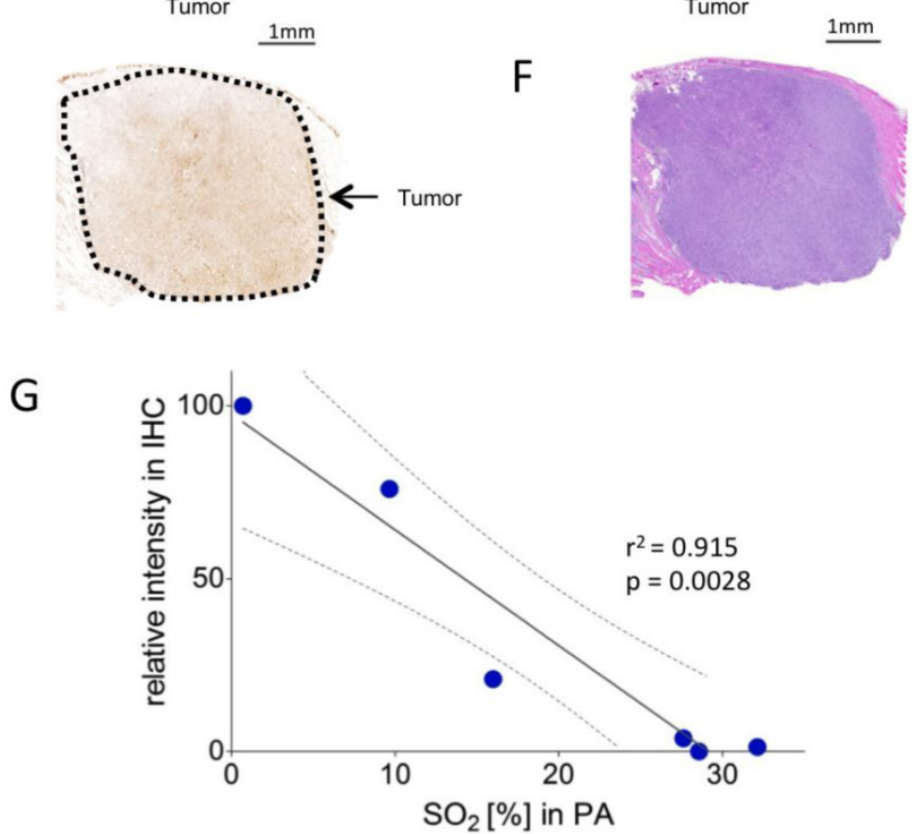

Figure 2. Assessment of tumor hypoxia in subcutaneous pancreatic tumor xenografts. A) Representative Oxy-Hemo PA image of a hypoxic s.c. tumor. B-mode image (left panel) and oxygen saturation map (Oxy-Hemo PA imaging, right panel) are placed side-by-side showing the same imaging window. The heat map represents oxygen saturation level, ranging from $100 \%$ (red) to $0 \%$ (dark blue). Spots below the defined threshold of total $\mathrm{Hb}(20 \%$ of the maximum intensity) are black (see methods). The yellow line represents the tumor region. B) Representative hypoxia staining (brown color) of the same s.c. tumor. The black line represents the tumor region. C) Representative H\&E staining of the hypoxic s.c. tumor displayed in Figure 2A. D) Representative Oxy-Hemo PA image of a non-hypoxic s.c. tumor. B-mode image and oxygen saturation map (Oxy-Hemo photoacoustic image is placed in the right panel showing the same imaging window). The heat map is as above. The yellow line represents the tumor region. $E$ ) Representative pimonidazole staining of the non-hypoxic s.c. tumor, which accordingly lacks brown staining, imaged in Figure 2D. The black line represents the tumor region. F) Representative H\&E staining of the same non-hypoxic s.c. tumor. G) Graphical representation of linear regression analysis from PA-based sO ${ }_{2}$ measurements and corresponding pimonidazole staining intensities. Pimonidazole intensities were normalized with the highest value in the data set adjusted to 100 and the lowest intensity set to 0 . Confidence bands for the $95 \%$-confidence interval are shown (dotted lines). 
To validate the correlation between PA in vivo oxygen saturation measurements and pimonidazole staining, PA measurements were correlated with normalized linear quantifications of pimonidazole stainings in six mice bearing subcutaneous KPC tumors.

Figure 2G shows a significant linear relation between $\mathrm{sO}_{2}$ as measured with PA and staining in-

\section{A}

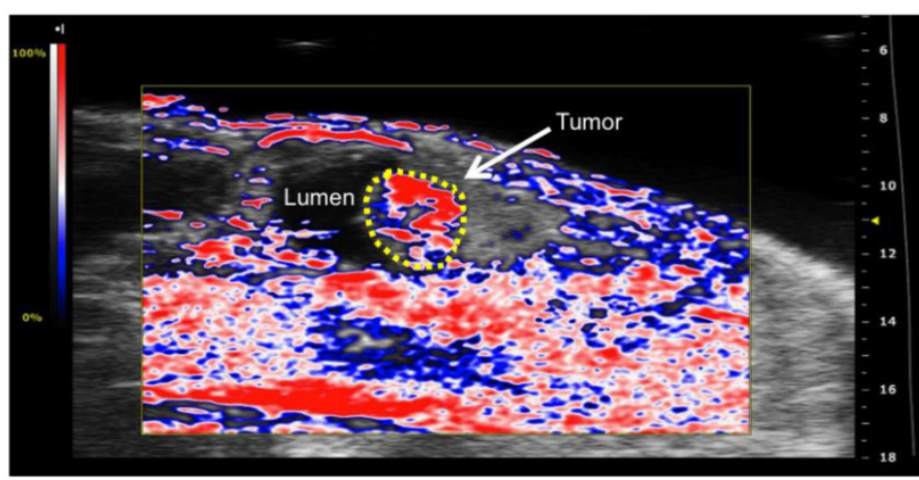

B

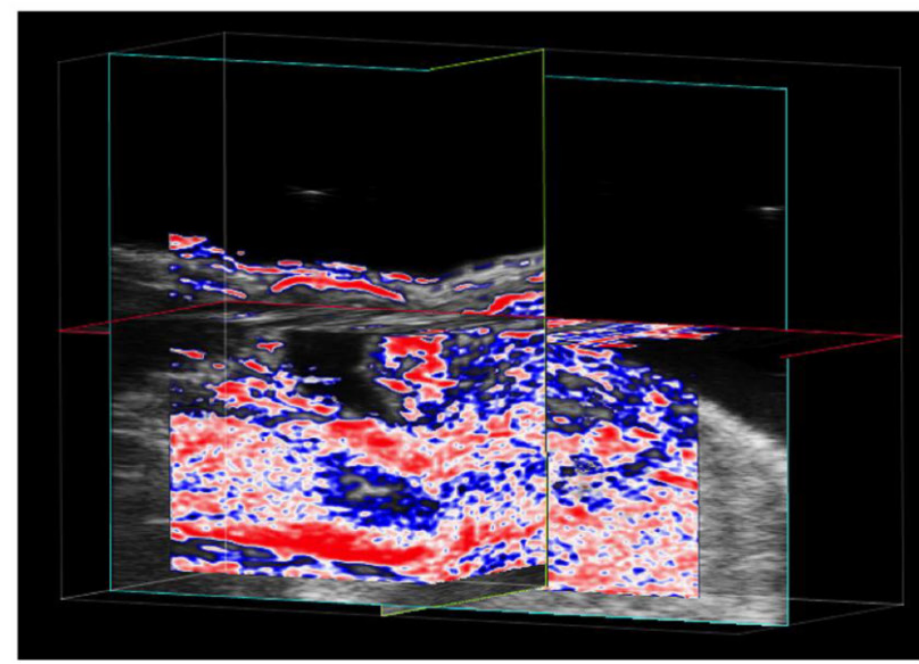

C

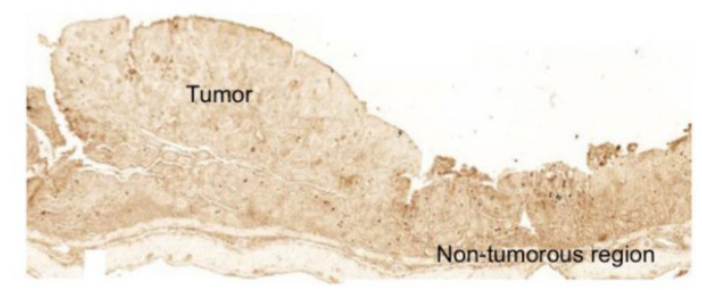

D

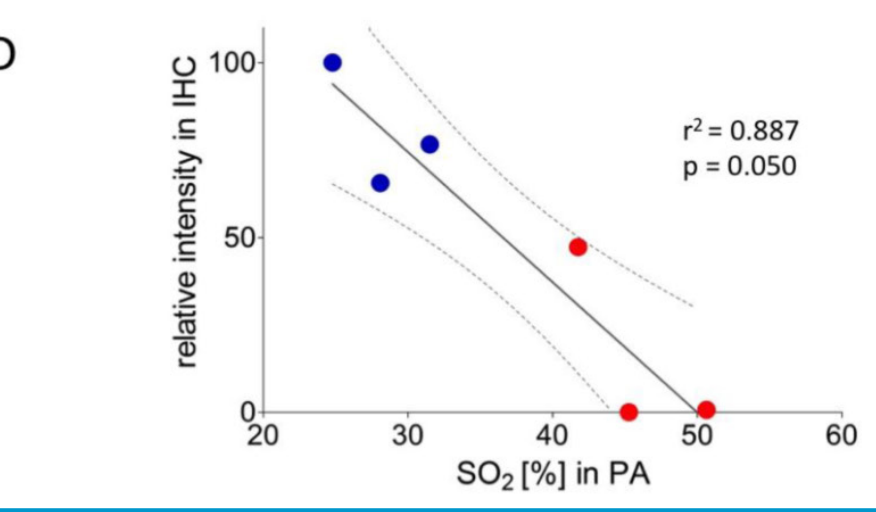

tensity $\left(\mathrm{r}^{2}=0.91, \mathrm{p}=0.0028\right)$.

Colon carcinoma was induced chemically by a single intraperitoneal administration of azoxymethane (AOM) followed by 3 cycles of dextran sulphate sodium (DSS) in drinking water, as described previously (28). The colonic tumors were scanned in B-mode as well as with PA. PA imaging showed well-oxygenized tumors in this model, positively correlating with immunohistochemical assessment of corresponding pimonidazole staining (Figure 3A-D).

\section{Photoacoustic imaging can be complemented by intravenous contrast to assess tumor perfusion}

From the previous experiments, the feasibility of real-time tissue hypoxia measurements was evident. Yet, PA measurements do not provide data on in vivo blood flow, which can be achieved by intravenous injection of US contrast medium. To complement PA analyses, we combined PA imaging with perfusion assessment through intravenously injected microbubbles (29). First, B-mode and PA images were acquired. Subsequently, microbubbles were injected i.v., and contrast enhancement was followed over time. Regions appearing similar in PA depicted different contrast kinetics, thereby providing a simultaneous and concise picture of oxygenation, perfusion and anatomical structure (Figure 4 and Additional file 2: Suppl. Video 2).

Figure 3. Oxygen saturation in a murine colon tumor. A) Representative Oxy-Hemo photoacoustic imaging of an oxygenized colon tumor. B-mode image overlaid with a PA-derived oxygen saturation map as in figure 2 . The yellow line represents the tumor region. B) Cross-view 3-dimensional representation of the same tumor, plains indicated in yellow, light blue, and red. C) Representative pimonidazole staining of the same oxygenized colorectal tumor as imaged in Figure 3A. D) Graphical representation of linear regression analysis from PA-based $\mathrm{sO}_{2}$ measurements and corresponding pimonidazole staining intensities. Pimonidazole intensities were normalized within the dataset as in figure 2 . Confidence bands for the $95 \%$-confidence interval are shown (dotted lines). Red dots represent tumor regions, blue dots represent adjacent non-tumorous regions. 
A
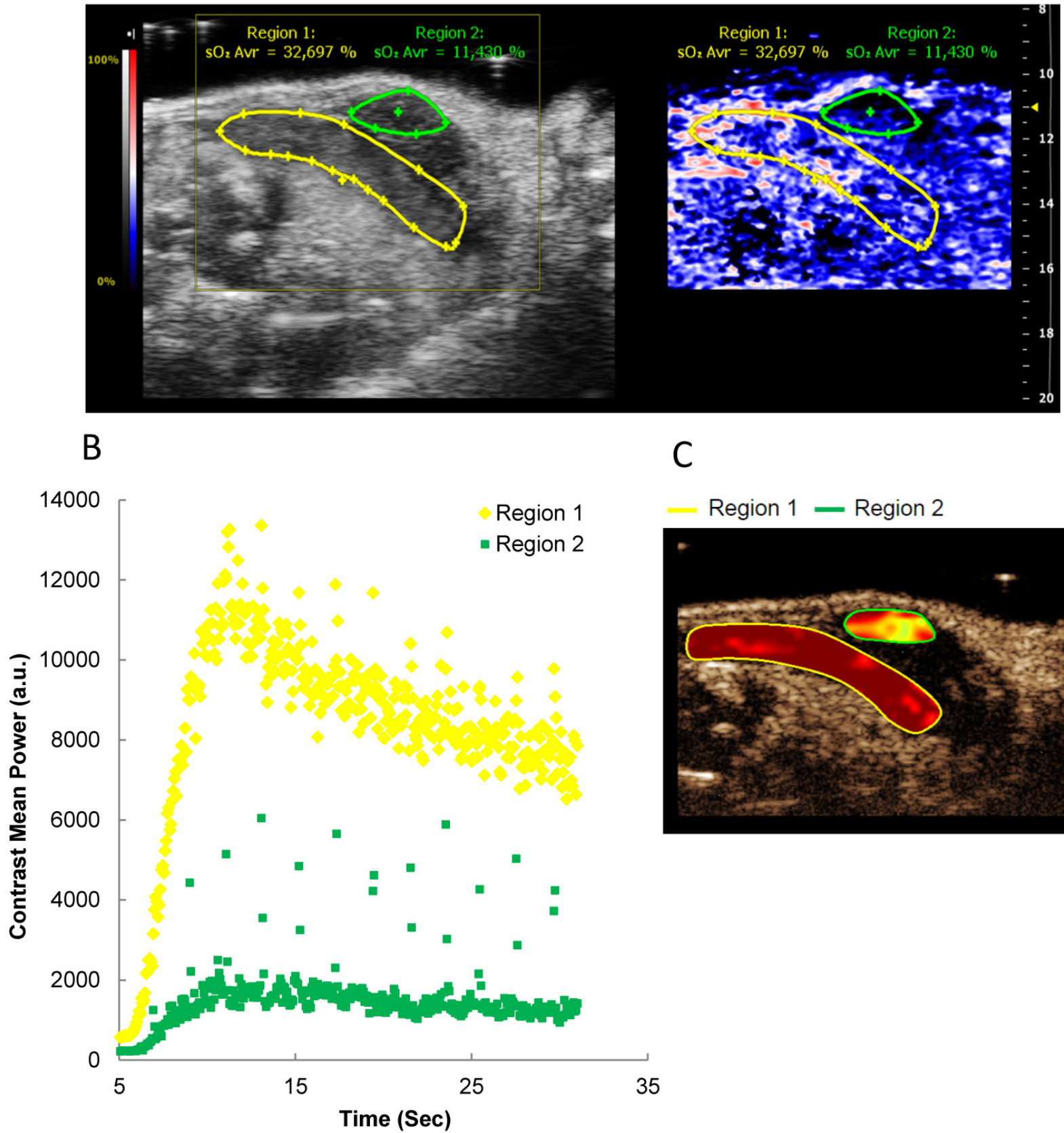

C

- Region 1 - Region 2

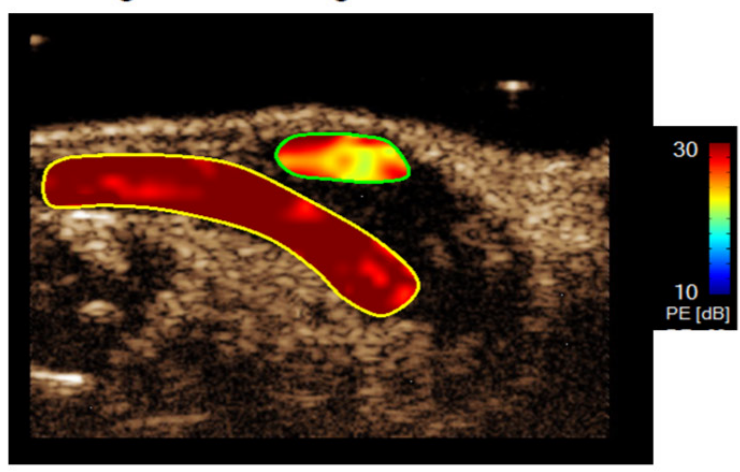

Figure 4. Oxygen saturation and tumor perfusion kinetics in a heterogeneous orthotopic pancreatic tumor. A) Representative Oxy-Hemo PA imaging of a heterogeneous orthotopic pancreatic cancer. B-mode image and oxygen saturation map (Oxy-Hemo photoacoustic imaging) are placed side-by-side showing the same imaging window. PA-derived oxygen saturation map is displayed as in figure 2. The green and yellow lines represent different tumor regions. Average $\mathrm{O}_{2}$ saturation levels are presented above the tumor regions in green and yellow, respectively. B) Microbubble wash-in curve of different tumor regions after bolus i.v. injection of microbubbles. C) Peak enhancement quantified by Vevo CQTM. The heat map represents contrast signal peak enhancement, range from 30 (red) to 10 (blue) $d B$. A higher tumor perfusion is observed in region I compared to region 2 .

\section{Discussion}

Major improvements in genetically engineered mice in combination with a diversity of emerging drug targets increase the need for preclinical imaging and longitudinal monitoring of treatment responses. Among the modalities used today are micro-computed tomography $(\mu \mathrm{CT})$ applications, magnetic resonance imaging (MRI), positron-emission tomography (PET), optical imaging modalities, and small-animal US $(30,31)$. PA imaging is complementary to this portfolio of modalities in that it can exploit endogenous contrast agents such as $\mathrm{HbO}_{2} / \mathrm{Hb}$ for non-invasive functional studies and offers the option of a combination with exogenous contrast agents (32).

Here, we present evidence that dual-wavelength-PA based on measurements of oxygenated and deoxygenated hemoglobin yields reliable 
real-time data of tissue oxygenation in mice and provide a positive correlation to histological analysis.

We first evaluated the feasibility of tissue saturation measurements by increasing inhaled oxygen saturation from ambient air $\left(21 \% \mathrm{O}_{2}\right)$ to $100 \%$, while monitoring saturation changes in real-time. As a proof of concept, supplying $100 \% \mathrm{O}_{2}$ led to a clear and reproducible rise in tissue oxygen saturation as measured with PA, which decreased accordingly when $\mathrm{O}_{2}$ supply was changed back to ambient levels.

Using the system (at $21 \% \mathrm{O}_{2}$ ) on s.c. pancreatic isografts in advanced stages demonstrated the ability of PA to assess tissue oxygenation in a murine tumor model. Other groups have used self-tailored PA devices to measure oxygenation in tumor tissue $(5,25)$ and were also able to show differences in tissue oxygenation in murine xenograft models, further advocating a role for PA to monitor functional aspects of tumorigenesis. In our study we could successfully correlate PA data with immunohistochemical hypoxia staining. In contrast to other recently published methods, dual-wavelength PA does not require exogenous contrast (25).

Oxygenation measurements with the described method rely on the presence of the endogenous chromophore, i.e. hemoglobin $(\mathrm{Hb})$. Areas with low levels of vascularization translating to low $\mathrm{Hb}$ concentrations will appear black in our figures, indicating that the concentration of $\mathrm{Hb}$ does not suffice to make a recordable measurement of oxygenation, or, alternatively, $\mathrm{Hb}$ is not present at significant levels because the tissue is necrotic or poorly vascularized. Therefore, distinguishing low-perfusion hypoxic areas and necrotic areas is challenging. To address this problem, we used non-targeted, air-filled microbubbles as systemic contrast agent in order to complement PA oxygenation measurements. The combination of conventional US, color- and power-doppler, dual-wave PA, and microbubbles can thus concisely address a multitude of aspects of vascularization, blood-flow, and tissue oxygenation; as such, bimodal imaging with PA and US can be considered a "one-stop-shop" application for longitudinal real-time imaging of vascularization and provide useful information in pharmaceutical studies that target the tumor vasculature (15).

Further clinical applications of PA are conceivable and currently being actively pursued: e.g. assessment of tumor oxygenation through a combination of endoscopic ultrasound and PA (12), or imaging of melanoma invasion and metastasis by exploiting melanin as an endogenous contrast medium, to name just two important examples. Targeted exogenous contrast agents will greatly broaden the spectrum of clinical applications (33).

Taken together, we provide evidence for the feasibility of real-time dual-wavelength PA imaging of preclinical murine cancer models to assess tissue hypoxia.

\section{Detailed Materials and Methods}

\section{Cells}

KPC cells were derived from the Kras ${ }^{L S L-G 12 D /+} ; p 5^{R 172 H /+} ; P d x 1-\underline{C}$ re mouse model for human pancreatic ductal adenocarcinoma (26). Cells were cultured in Dulbecco's modified Eagle's medium (DMEM)/F12 (1:1 mixture) (Gibco Life Technologies Europe BV), supplemented with $10 \%$ fetal bovine serum (Gibco Life Technologies Europe BV), 100 $\mathrm{U} / \mathrm{ml}$ penicillin, $100 \mathrm{mg} / \mathrm{ml}$ streptomycin (Gibco Life Technologies Europe BV), at $37^{\circ} \mathrm{C}$ and $5 \% \mathrm{CO}_{2}$. Fibroblasts were generated from pancreata of 8 to 10 weeks old C57Bl/6J mice. Isolated pancreata were minced, incubated at $37^{\circ} \mathrm{C}$ in $3 \mathrm{ml}$ HBSS (Gibco Life Technologies Europe BV) with $1 \mathrm{mg} / \mathrm{ml}$ CollagenaseP (Roche, Bromma, Sweden) for 40 minutes. $1 \mathrm{ml}$ DNAse $(1 \mathrm{mg} / \mathrm{ml}$; Roche, Bromma, Sweden) was added, gently mixed and specimens were centrifuged. The cell pellet was washed once with HBSS, resuspended in DMEM/F12 (Gibco Life Technologies Europe $\mathrm{BV}$ ) containing $10 \%$ fetal calf serum (FCS) plus antibiotics as above, and plated on $1 \%$ gelatin (VWR, Stockholm, Sweden) coated T75 cell culture flasks (TPP, Nordic biolabs, Sweden). The primary cultures were expanded four times before freezing in liquid nitrogen. Cells were thawed and cultured for two days before injection.

\section{Animals, tumor inoculation and tumor induction}

Ethical permission for animal experiments was obtained from the local ethics committee (No. S89/12, $\mathrm{S} 69 / 12$, and S38/13, plus extensions). C57Bl/6J mice were obtained from Scanbur, Sweden, and used for all experiments at 6- 8 weeks of age.

Subcutaneous pancreatic tumor xenografts $(\mathrm{n}=$ 6 , female) were established by s.c. injection of $2.5 \times 10^{5}$ KPC cells mixed with $7.5 \times 10^{5}$ murine fibroblasts in a total volume of $50 \mathrm{ul}$ phosphate buffered saline. Two weeks after tumor inoculation, mice were scanned using high-frequency US as described below.

For intrapancreatic tumor injections, mice $(n=3$, female) were anesthetized via intra-peritoneal injection of Ketamine $80 \mathrm{mg} / \mathrm{kg}$ and Xylazine $8 \mathrm{mg} / \mathrm{kg}$. Abdominal hair was removed (Aesculap Exacta rodent shaver, Agnthos, Sweden) followed by skin disinfection with Jodopax-Vet diluted 1:25 in deionized water, over the left flank. Anesthetized mice were placed on a heating pad $\left(40^{\circ} \mathrm{C}\right)$ during the remainder of the procedure until post-operative awakening. A 
left subcostal incision was made, and the pancreas and the spleen were gently exteriorized. Then, $1 \times 10^{6}$ KPC cells suspended in in a total volume of $50 \mathrm{ul}$ phosphate buffered saline were orthotopically injected into the pancreatic tail using a 26 gauge needle (0.45 x $10 \mathrm{~mm})$ (Becton Dickinson Medical, Sweden). After injection, the needle was kept in position for $10 \mathrm{~s}$ before being slowly removed. The pancreas and the spleen were returned into the abdomen, and the wound was closed using interrupted 6-0 sutures (absorbable synthetic, Ethicon, LLC, Agnthos, Sweden). Mice received pain relief with buprenorphine at 0.06 $\mathrm{mg} / \mathrm{kg}$ for three consecutive days and $500 \mu \mathrm{l}$ saline to restore the fluid balance, both s.c.. Eleven days after tumor inoculation, mice were scanned as described below.

For the model of colitis-associated cancer, azoxymethane was injected i.p. (12.5 mg/kg, Sigma-Aldrich) at day 0 , followed by 3 cycles of $2 \%$ dextran sulphate sodium in drinking water (TdB consultancy, Uppsala, Sweden) for 5 days, with the first cycle starting at day 5 , followed by 14 days intervals with regular drinking water (34). Mice were imaged one week after the third course of DSS ( $n=6$ mice).

\section{High-frequency ultrasound and photoacoustic imaging}

All US and PA scanning was performed on a VisualSonics Vevo 2100 LAZR Imaging Station (VisualSonics, Inc., Toronto, Canada) (15). Mice were anesthetized using $1.5 \%$ isoflurane with medical air at a flow of $2 \mathrm{~L} / \mathrm{min}$. Hair was removed over areas of interest using a depilatory cream. US gel (Parker Laboratories Inc., USA) was applied over the region of interest. For transcutaneous colon cancer imaging, the murine colonic lumen was gently inflated with US gel. During image acquisition, ECG, respiration, blood pressure, and body temperature were monitored, and anesthesia depth adjusted if necessary.

To collect anatomical information at high resolution, B-mode imaging was acquired using a high-frequency ultrasound probe (MS550D, VisualSonics, Canada, broadband frequency: $22 \mathrm{MHz}$ $55 \mathrm{MHz}$, image axial resolution: $40 \mathrm{um}$ ) at $40 \mathrm{MHz}$. A previously published manual was used for anatomical reference in pancreatic imaging (35).

Blood flow in small blood vessels was imaged using Power-Doppler mode at $16 \mathrm{MHz}, 100 \%$ power, using an LZ250 transducer (VisualSonics, Canada, broadband frequency: $13 \mathrm{MHz}-24 \mathrm{MHz}$,image axial resolution: $75 \mu \mathrm{m}$; data not shown).

During PA imaging, a tunable laser (680-970 nm) was used as described previously (15). Pulse-to-pulse energy fluctuation was continuously monitored, and variations exceeding $25 \%$ lead to recalibration of the system before further imaging. Oxygen saturation and hemoglobin concentration were measured at 21 $\mathrm{MHz}$ frequency (LZ250, VisualSonics, Canada). In Oxy-Hemo mode, PA dual-wavelength imaging at 750 and $850 \mathrm{~nm}$ creates images of oxygenated hemoglobin and deoxygenated hemoglobin, which are co-registered with grey scale B-mode imaging (detailed description of algorithms in (15)). Within a region of interest (ROI), oxygen saturation $\left(\mathrm{SO}_{2} \%\right)$ was calculated as the percentage of oxygenated hemoglobin in total hemoglobin. Quantification of total hemoglobin and oxygen saturation were achieved using the HemoMeaZure ${ }^{\mathrm{TM}}$ tool (VisualSonics, Canada) and OxyZated $^{\mathrm{TM}}$ tool (VisualSonics, Canada), respectively. Low total $\mathrm{Hb}$ levels (e.g. in areas with low perfusion and absence of big vessels) result in noise-relatedly unreliable saturation measurements. As a means of noise-control, the system used offers the possibility to discard levels below a defined threshold of total $\mathrm{Hb}$ in relation to the maximum possibly level. This threshold was set to $20 \%$ for all experiments as this was found to represent an empirically reasonable value. All spots below the threshold will appear black in all images.

Nonlinear contrast imaging was acquired using the LZ250 transducer at a frequency of $18 \mathrm{MHz}$, power of $4 \%$, and a contrast gain of $25 \mathrm{~dB}$, immediately after an intravenous bolus injection of $50 \mu \mathrm{L}$ non-targeted microbubbles (VevoMicroMarker Contrast Agent, VisualSonics, Canada) at the concentration of $2 \times 10^{9} / \mathrm{ml}$ (36). A cine loop was recorded at a frame rate of $15 / \mathrm{sec}$. Tumor perfusion and peak enhancement at different ROIs were quantified with VevoCQ ${ }^{\mathrm{TM}}$ Software (VisualSonics, Canada), as described previously (37).

3D-mode imaging was acquired via a 3D acquisition motor scanned along the vertical axis. 3D volumetric data was generated by integration of multiple two-dimensional US images (z-axis step size of 0.5 $\mathrm{mm})$.

\section{Immunohistochemistry and quantification}

To visualize hypoxic areas by immunohistochemistry, a commercially available hypoxyprobe kit (Hypoxyprobe $^{\mathrm{TM}}-1$ Omni, Hypoxyprobe, Inc, USA) was utilized as previously described (38). Briefly, pimonidazole was intraperitoneally injected at a dose of $60 \mathrm{mg} / \mathrm{kg}$ body weight $1 \mathrm{~h}$ before the mice were euthanized. Tumors were harvested, fixed in $4 \%$ formaldehyde overnight, and processed for paraffin sectioning. Immunohistochemistry staining against pimonidazole protein adducts was performed using VECTASTAIN ABC kit (Vector laboratories, USA) according to the manufacturer's protocol. Briefly, deparaffinized tissue sections were incubated with 
$0.3 \% \mathrm{H}_{2} \mathrm{O}_{2}$ for 30 minutes to block endogenous peroxidase activity. The tissue sections were then incubated in diluted normal goat serum for $1 \mathrm{~h}$ to block non-specific binding sites. The rabbit anti-pimonidazole antibody (Hypoxyprobe, Inc, USA, 1:100) was applied overnight at $4{ }^{\circ} \mathrm{C}$. After three times washing with Tris-Buffered Saline Tween-20, slides were incubated with the secondary antibody, biotinylated goat anti-rabbit IgG (Vector laboratories, USA, 1:200) for $1 \mathrm{~h}$ at room temperature. After incubation with VECTASTAIN ABC Reagent for 30 minutes, hypoxia staining was developed by DAB substrate (Vector laboratories, USA) and nuclei were counterstained by hematoxylin. ImageJ software (v 1.46r, U.S. National Institutes of Health, Bethesda, MD, USA) was used to quantify brown color intensity over bright field microscopic images. Intensity histograms of pimonidazole were acquired with ImageJ from whole tumors corresponding to areas measured with PA. Histogram values were averaged and intensities over the dataset were normalized to correspond to relative intensities ranging from 0 to 100 (separately for each dataset in figures 2 and 3). Data were analyzed together with corresponding saturation values of PA measurements using GraphPad Prism v5.01 (La Jolla, CA, USA).

\section{Supplementary Material}

Additional File 1:

Suppl. video 1.

http://www.thno.org/v04p0604s1.gif

Additional File 2:

Suppl. video 2.

http://www.thno.org/v04p0604s2.gif

\section{Acknowledgements}

MG received a postdoctoral stipend from the German Research Foundation (DFG Ge 2386/1-1). This work was supported by a seed grant from the Strategic Alliance for Cancer Research (StratCan) to YZ from the preclinical cancer test facility (PCTF). Further support was received from the Swedish Research Council (to JML Vetenskapsrådet, No. K2013-67X-22322-01-3) and the Swedish Cancer Society (to RH, Cancerfonden, No. 120771 \& 100282). $\mathrm{BE}$ was supported by an MD fellowship of the Boehringer Ingelheim Fonds. AN was supported by a Mildred Scheel postdoctoral fellowship from the German Cancer Aid. MH gratefully received funding from the Swedish Cancer Society (Cancerfonden) and the Swedish Children Cancer Association (Barncancerfonden). We are grateful to Rune Toftgård for advice on the manuscript, Elin Tüksammel for technical assistance in the animal facility, and Annika Wagman for assistance with cell culture and com- ments on the manuscript. We thank the Visualsonics team for technical assistance in setting up the imaging system. The Vevo 2100 US system was acquired with grant support from the Jonasson Center for Medical Imaging, Huddinge, Sweden.

\section{Competing Interests}

The authors have declared that no competing interest exists.

\section{References}

1. Wang LV, Hu S. Photoacoustic Tomography: In Vivo Imaging from Organelles to Organs. Science. 2012 Mar 23;335(6075):1458-62.

2. Wu L, Cai X, Nelson K, Xing W, Xia J, Zhang R, et al. A Green Synthesis of Carbon Nanoparticle from Honey for Real-Time Photoacoustic Imaging. Nano Res. 2013;6(5):312-25

3. Hu X, Wei C-W, Xia J, Pelivanov I, O'Donnell M, Gao X. Nanoparticles: Trapping and Photoacoustic Detection of CTCs at the Single Cell per Milliliter Level with Magneto-Optical Coupled Nanoparticles (Small 12/2013). Small. 2013 Jun 24;9(12):2045.

4. Lin J, Wang S, Huang P, Wang Z, Chen S, Niu G, et al. Photosensitizer-loaded gold vesicles with strong plasmonic coupling effect for imaging-guided photothermal/photodynamic therapy. ACS Nano. 2013 Jun 25;7(6):5320-9.

5. Chen Z, Yang S, Xing D. In vivo detection of hemoglobin oxygen saturation and carboxyhemoglobin saturation with multiwavelength photoacoustic microscopy. Opt Lett. 2012 Aug 15;37(16):3414-6.

6. Hysi E, Saha RK, Kolios MC. Photoacoustic ultrasound spectroscopy for assessing red blood cell aggregation and oxygenation. J Biomed Opt. 2012 Dec;17(12):125006

7. Rowland KJ, Yao J, Wang L, Erwin CR, Maslov KI, Wang LV, et al. Up-regulation of hypoxia-inducible factor 1 alpha and hemodynamic responses following massive small bowel resection. J Pediatr Surg. 2013 Jun;48(6):1330-9.

8. Horecker BL. The Absorption Spectra of Hemoglobin and Its Derivatives in the Visible and Near Infra-Red Regions. J Biol Chem. 1943 Apr 1;148(1):173-83.

9. Oladipupo S, Hu S, Kovalski J, Yao J, Santeford A, Sohn RE, et al. VEGF is essential for hypoxia-inducible factor-mediated neovascularization but dispensable for endothelial sprouting. PNAS. 2011 Aug 9;108(32):13264-9.

10. Yao J, Maslov KI, Zhang Y, Xia Y, Wang LV. Label-free oxygen-metabolic photoacoustic microscopy in vivo. J Biomed Opt. 2011 Jul 1;16(7):076003-076003.

11. Laufer J, Johnson P, Zhang E, Treeby B, Cox B, Pedley B, et al. In vivo preclinical photoacoustic imaging of tumor vasculature development and therapy. J Biomed Opt. 2012 May 21;17(5):056016-1.

12. Yang J-M, Favazza C, Chen R, Yao J, Cai X, Maslov K, et al. Simultaneous functional photoacoustic and ultrasonic endoscopy of internal organs in vivo. Nature Medicine. 2012;18(8):1297-302.

13. Sun $Y$, Stephens DN, Park J, Sun Y, Marcu L, Cannata JM, et al. Development of a Multi-modal Tissue Diagnostic System Combining High Frequency Ultrasound and Photoacoustic Imaging with Lifetime Fluorescence Spectroscopy. Proc IEEE Ultrason Symp. 2008::570-3.

14. Hsieh B-Y, Chen S-L, Ling T, Guo LJ, Li P-C. All-optical scanhead for ultrasound and photoacoustic dual-modality imaging. Opt Express. 2012 Jan 16;20(2):1588-96.

15. Needles A, Heinmiller A, Sun J, Theodoropoulos C, Bates D, Hirson D, et al Development and initial application of a fully integrated photoacoustic micro-ultrasound system. IEEE Transactions on Ultrasonics, Ferroelectrics and Frequency Control. 2013;60(5):888-897.

16. Pouysségur J, Dayan F, Mazure NM. Hypoxia signalling in cancer and approaches to enforce tumour regression. Nature. 2006 May 25;441(7092):437-43.

17. Vaupel P, Mayer A. Hypoxia in cancer: significance and impact on clinical outcome. Cancer Metastasis Rev. 2007 Jun 1;26(2):225-39.

18. Meijer TWH, Kaanders JHAM, Span PN, Bussink J. Targeting hypoxia, HIF-1, and tumor glucose metabolism to improve radiotherapy efficacy. Clin Cancer Res. 2012 Oct 15;18(20):5585-94.

19. Rapisarda A, Melillo G. Overcoming disappointing results with antiangiogenic therapy by targeting hypoxia. Nat Rev Clin Oncol. 2012 Jul;9(7):378-90.

20. Washington MK, Powell AE, Sullivan R, Sundberg JP, Wright N, Coffey RJ, et al. Pathology of Rodent Models of Intestinal Cancer: Progress Report and Recommendations. Gastroenterology. 2013 Apr;144(4):705-17.

21. Zhang Y, Chen L, Yang J, Fleming JB, Chiao PJ, Logsdon CD, et al. Study human pancreatic cancer in mice: how close are they? Biochim Biophys Acta. 2013 Jan;1835(1):110-8.

22. Langdon SP. Animal modeling of cancer pathology and studying tumor response to therapy. Curr Drug Targets. 2012 Nov;13(12):1535-47.

23. Meng-Lin Li, Jung-Taek Oh, Xueyi Xie, Geng Ku, Wei Wang, Chun Li, et al. Simultaneous Molecular and Hypoxia Imaging of Brain Tumors In Vivo Using Spectroscopic Photoacoustic Tomography. Proceedings of the IEEE. 2008 Mar;96(3):481-9. 
24. Lungu GF, Li M-L, Xie X, Wang LV, Stoica G. In vivo imaging and characterization of hypoxia-induced neovascularization and tumor invasion. International Journal of Oncology. 2007 Jan 1;30(1):45-54.

25. Shao Q, Morgounova E, Jiang C, Choi J, Bischof J, Ashkenazi S. In vivo photoacoustic lifetime imaging of tumor hypoxia in small animals. J Biomed Opt. 2013 Jul;18(7):076019.

26. Neesse A, Frese KK, Bapiro TE, Nakagawa T, Sternlicht MD, Seeley TW, et al. CTGF antagonism with mAb FG-3019 enhances chemotherapy response without increasing drug delivery in murine ductal pancreas cancer. PNAS. 2013 Jul 23;110(30):12325-30.

27. Denekamp J, Michael BD, Minchinton AI, Smithen CE, Stewart FA, Stratford $\mathrm{MR}$, et al. Comparative studies of hypoxic-cell radiosensitization using artificially hypoxic skin in vivo. Br J Cancer. 1982 Feb;45(2):247-55.

28. Gerling M, Glauben R, Habermann JK, Kühl AA, Loddenkemper C, Lehr H-A, et al. Characterization of Chromosomal Instability in Murine Colitis-Associated Colorectal Cancer. PLoS ONE. 2011 Jul 22;6(7):e22114.

29. Horie S, Chen R, Li L, Mori S, Kodama T. Contrast-enhanced high-frequency ultrasound imaging of early stage liver metastasis in a preclinical mouse model. Cancer Letters. Available from: http://www.sciencedirect.com/science/article/pii/S0304383513004564

30. Albanese C, Rodriguez OC, VanMeter J, Fricke ST, Rood BR, Lee Y, et al. Preclinical magnetic resonance imaging and systems biology in cancer research: current applications and challenges. Am J Pathol. 2013 Feb;182(2):312-8.

31. Koba W, Jelicks LA, Fine EJ. MicroPET/SPECT/CT Imaging of Small Animal Models of Disease. The American Journal of Pathology. 2013 Feb;182(2):319-24.

32. Garcia-Allende PB, Glatz J, Koch M, Ntziachristos V. Enriching the interventional vision of cancer with fluorescence and optoacoustic imaging. J Nucl Med. 2013 May;54(5):664-7.

33. Liu X, Lee C, Law W-C, Zhu D, Liu M, Jeon M, et al. Au-Cu2-xSe Heterodimer Nanoparticles with Broad Localized Surface Plasmon Resonance as Contrast Agents for Deep Tissue Imaging. Nano Lett. 2013.

34. Glauben R, Batra A, Stroh T, Erben U, Fedke I, Lehr HA, et al. Histone Deacetylases: Novel Targets for Prevention of Colitis-Associated Cancer in Mice. Gut. 2008 May 1;57(5):613-22.

35. Sastra SA, Olive KP. Quantification of Murine Pancreatic Tumors by High-Resolution Ultrasound. In: Su GH, editor. Pancreatic Cancer [Internet]. Humana Press; 2013; $\quad 249-66 . \quad$ Available from: http://link.springer.com/protocol/10.1007/978-1-62703-287-2_13

36. Olson P, Chu GC, Perry SR, Nolan-Stevaux O, Hanahan D. Imaging guided trials of the angiogenesis inhibitor sunitinib in mouse models predict efficacy in pancreatic neuroendocrine but not ductal carcinoma. Proc Natl Acad Sci USA. 2011 Dec 6;108(49 E): 1275-1284.

37. Bachawal SV, Jensen KC, Lutz AM, Gambhir SS, Tranquart F, Tian L, et al. Earlier Detection of Breast Cancer with Ultrasound Molecular Imaging in a Transgenic Mouse Model. Cancer Res. 2013 Mar 15;73(6):1689-98.

38. Mastrogiannaki M, Matak P, Keith B, Simon MC, Vaulont S, Peyssonnaux C. HIF-2a, but not HIF-1a, promotes iron absorption in mice. Journal of Clinical Investigation. 2009 May 1;119(5):1159-66. 